Proceedings of the Edinburgh Mathematical Society (2007) 50, 389-409 (C)

DOI:10.1017/S001309150500101X Printed in the United Kingdom

\title{
ON THE BLOW-UP OF THE NON-LOCAL THERMISTOR PROBLEM
}

\author{
N. I. KAVALLARIS ${ }^{1,2}$ AND T. NADZIEJA ${ }^{2}$ \\ ${ }^{1}$ Instytut Matematyczny, Uniwersytet Wroctawski, \\ pl. Grunwaldzki 2/4, 50-384 Wroctaw, Poland \\ ${ }^{2}$ Wydziat Matematyki, Informatyki i Ekonometrii, Uniwersytet Zielonogórski, \\ ul. Szafrana 4a, 65-516 Zielona Góra, Poland \\ (nkaval@math.ntua.gr; t.nadzieja@wmie.uz.zgora.pl)
}

(Received 14 July 2005)

Abstract The conditions under which the solution of the non-local thermistor problem

$$
\begin{gathered}
u_{t}=\Delta u+\frac{\lambda f(u)}{\left(\int_{\Omega} f(u) \mathrm{d} x\right)^{2}}, \quad x \in \Omega \subset \mathbb{R}^{N}, N \geqslant 2, t>0, \\
\frac{\partial u(x, t)}{\partial \nu}+\beta(x) u(x, t)=0, \quad x \in \partial \Omega, t>0, \\
u(x, 0)=u_{0}(x), \quad x \in \Omega,
\end{gathered}
$$

blows up are investigated. We assume that $f(s)$ is a decreasing function and that it is integrable in $(0, \infty)$. Considering a suitable functional we prove that for all $\lambda>0$ the solution of the Neumann problem blows up in finite time. The same result is obtained for the Robin problem under the assumption that $\lambda$ is sufficiently large $(\lambda \gg 1)$. In the proof of existence of blow-up for the Dirichlet problem we use the subsolution technique. We are able to construct a blowing-up lower solution under the assumption that either $\lambda>\lambda^{*}$ or $0<\lambda<\lambda^{*}$, for some critical value $\lambda^{*}$, and that the initial condition is sufficiently large provided also that $f(s)$ satisfies the decay condition $\int_{0}^{\infty}\left[s f(s)-s^{2} f^{\prime}(s)\right] \mathrm{d} s<\infty$.

Keywords: non-local parabolic problems; blow-up; comparison techniques; thermistor problem

2000 Mathematics subject classification: Primary 35K60; 35B50; 35Q

Secondary 58J90; 58J35

\section{Introduction}

The operation of the thermistor, a device for regulating electric current in a circuit, is described by the following system:

$$
\begin{gathered}
u_{t}=\nabla \cdot(\kappa(u) \nabla u)+\rho(u)|\nabla \phi|^{2}, \quad x \in \Omega, t>0, \\
\nabla \cdot(\rho(u) \nabla \phi)=0, \quad x \in \Omega, t>0,
\end{gathered}
$$

together with some boundary conditions for $u(x, t)$ and $\phi(x, t)$ on $\partial \Omega$ (see, for example, $[3,4]) . \Omega$ is assumed to be a smooth, bounded open set of $\mathbb{R}^{N}, N \geqslant 1$, and stands for the spatial domain occupied by the conductor (the body of the thermistor); the physical 
situation corresponds to $N=3$. Moreover, $\phi(x, t)$ is the electrical potential, $u(x, t)$ the temperature inside the conductor, $\kappa(u)>0$ the thermal conductivity, and $\rho(u)>0$ stands for the electrical conductivity. The parabolic equation (1.1) describes the heat flow in the system, while the elliptic equation (1.2) describes the conservation of charge in the system provided that its variation in space and time is not too rapid.

Under some simplifications the system (1.1), (1.2) might be reduced to a single, but non-local, equation. Let us suppose that the thermal conductivity $\kappa$, the density and the specific heat of the conductor are constant. If in addition the initial distribution of temperature changes only in the direction of the $x$-axis and the potential difference $V$ applied on the sides of the conductor is fixed, then system (1.1), (1.2) after scaling takes the form of a one-dimensional single equation:

$$
u_{t}=u_{x x}+\frac{\lambda f(u)}{\left(\int_{-1}^{1} f(u) \mathrm{d} x\right)^{2}}, \quad-1<x<1, t>0,
$$

where $f(u)$ stands for the electrical resistance of the conductor and $\lambda=V^{2}$ is the parameter of the problem. The same one-dimensional, non-local model describes the variation of temperature in the case where the conductor-thermistor is short and fat. In contrast with this one-dimensional model, the second configuration, when the conductorthermistor is long and thin, is described by a two-dimensional model:

$$
u_{t}=\Delta u+\frac{\lambda f(u)}{\left(\int_{D} f(u) \mathrm{d} x\right)^{2}}, \quad x \in D, t>0, D \subset \mathbb{R}^{2},
$$

where $D$ is the cross-section of the conductor-thermistor $\Omega, f(u)$ now represents the electrical conductivity of the conductor, and $\lambda=I^{2} / A^{2}$ with $A$ the area of the crosssection $D$. In this case, the current $I$ is considered to be constant. According to the nature of the conductor, the resistivity may be either an increasing or decreasing function of temperature. Consequently, $f$ could be assumed to be a monotone function. For a detailed analysis regarding the construction of the models (1.3) and (1.4) see $[\mathbf{2 0}, \mathbf{2 3}]$ and the references therein. For another configuration of the conductor-thermistor, the so-called narrowing process, which considers that only a small part, and not the whole of the thermistor, is cylindrical, see [5]. Also, steady states of the full problem (1.1), (1.2) were investigated in $[\mathbf{1}, \mathbf{1 0}, \mathbf{1 1}]$.

Equation (1.3) can also be used to describe thermoviscous flow of linear materials. In this case, stress $\tau$ is assumed to satisfy $\partial \tau / \partial x=0$ and $\tau \propto \partial v / \partial x$, where $v(x, t)$ is the velocity in the $y$-direction. For a nonlinear material the same situation is described, after some scaling, by the equation

$$
u_{t}=u_{x x}+\frac{\lambda f(u)}{\left(\int_{-1}^{1} f(u) \mathrm{d} x\right)^{p}}, \quad-1<x<1, t>0,
$$

where $p>1$, since now $\tau \propto(\partial v / \partial x)^{k}$ for some $k>0$ (see [22]). In the case of a nonlinear conductor, when $|j| \propto|\nabla \phi|^{k}, k>0$, a two-dimensional version

$$
u_{t}=\Delta u+\frac{\lambda f(u)}{\left(\int_{\Omega} f(u) \mathrm{d} x\right)^{p}}, \quad x \in \Omega, t>0, \Omega \subset \mathbb{R}^{2},
$$


$p>1$, can be derived to describe the thermoelectric flow in the conductor (see $[\mathbf{2 2}]$ and the references therein).

Also, for $p=1$ equation (1.5) can describe phenomena associated with the occurrence of shear bands in metals being deformed under high strain rates $\left[\mathbf{6}_{-\mathbf{8}} \mathbf{8}\right]$, in the theory of gravitational equilibrium of polytropic stars [19], in the investigation of the fully turbulent behaviour of real flows, using invariant measures for the Euler equation [9], and in modelling aggregation of cells via interaction with a chemical substance (chemotaxis) $[\mathbf{2 4}]$.

In this work we do not dwell on existence and uniqueness results. We always assume that our equation (1.4), with some boundary and initial conditions, has a unique sufficiently smooth solution in a small time interval $(0, T)$. Such (local) existence and uniqueness results, for a Lipschitz function satisfying $f(s) \geqslant c>0, s>0$, can be derived using Picard iteration type arguments (see [20]). Moreover, these arguments also yield that the solution exists as long as it remains bounded, i.e. solutions cease to exist only through blow-up $\left(\|u(\cdot, t)\|_{\infty} \rightarrow \infty\right.$ as $\left.t \rightarrow t^{*} \leqslant \infty\right)$. With the same arguments we can also prove local existence and uniqueness in the case of a temperature-dependent thermal conductivity $\kappa(u)>0$ (see problems (2.10)-(2.12) and (3.8)-(3.10) below). For some blow-up results regarding the case when $p=1$, see $[\mathbf{1 6}, \mathbf{2 4}]$.

The question of the occurrence of blow-up for the thermistor problem has attracted the interest of many researchers. First Antontsev and Chipot suggested in [3], and then improved in [4], an energy method to prove blow-up for the solution of the system (1.1), (1.2). Barabanova [5], using the same method, proved blow-up for the solution of the system (1.1), (1.2), considering a special configuration of the problem (see above). Considering now the non-local problem (1.2), $N>1$, if $f$ is a decreasing function, we have a variation of the comparison results that apply to the more standard parabolic problems (see $[\mathbf{2 0}]$ ). Taking advantage of this fact, Lacey $[\mathbf{2 0}, \mathbf{2 1}]$ and Tzanetis $[\mathbf{2 3}]$ proved the occurrence of blow-up for the one-dimensional model (1.3) and for the two-dimensional radially symmetric model (1.4), respectively. Actually, first they estimated the supremum $\lambda^{*}$ of the spectrum of the related steady-state problem and then they proved blow-up, for $\lambda>\lambda^{*}$, by constructing some blowing-up lower solutions for the problem. If $f$ is an increasing function, then blow-up cannot take place, as has been proven in $[\mathbf{6}, \mathbf{2 0}]$. In this case, blow-up would be expected to occur only if $p<1[\mathbf{6}, \mathbf{1 7}]$.

In this article, we generalize the blow-up results existing in $[\mathbf{2 0 , 2 1 , 2 3 ]}$, for dimensions $N \geqslant 2, p>1$, and for nonlinear-diffusion non-local problems. More precisely, for Neumann and Robin boundary conditions, using the energy method introduced in $[\mathbf{3}, \mathbf{4}]$, we prove that in the case of a rapidly decreasing $f$, blow-up occurs for every $\lambda>0$ and for sufficiently large $\lambda$, respectively. The main advantage of this method is that it does not require any knowledge about the corresponding steady-state problem, while for the methods used in $[\mathbf{2 0}, \mathbf{2 1}, \mathbf{2 3}]$ such a knowledge was necessary. Moreover, it can be used to prove the occurrence of blow-up in the case of a nonlinear-diffusion ohmic heating process and for $p>1$ as well. This method provides us, in the case of the Robin problem, with an upper bound for the supremum $\lambda^{*}$ of the spectrum of the associated steady-state problem. Unfortunately, this method does not seem to be applicable for Dirichlet boundary 
conditions. In this case, we again prove blow-up but using comparison techniques. Using some ideas of Bebernes and Lacey $[\mathbf{6}]$, we construct, for large enough initial conditions, a lower solution that blows up. This construction provides us with an upper estimate of the blow-up time with respect to $\lambda-\lambda^{*}$ that seems to be in agreement with the blow-up estimates given in $[\mathbf{1 8}]$ for the Robin problem. In any case we prove that blow-up is global and uniform (flat), i.e. $u(x, t) \sim\|u(\cdot, t)\|_{\infty}$ as $t \rightarrow t^{*}-$ for a.e. $x \in \Omega$.

The plan of the paper is as follows. In $\S 2$, we study the Neumann problem. We construct a proper functional which allows us to deduce finite blow-up for the solution $u(x, t)$ and for every $\lambda>0$. In $\S 3$, modifying the form of the functional constructed in $\S 2$, we prove blow-up for the solution of the Robin problem for large values of the parameter $\lambda$. Finally, in $\S 4$ we consider the Dirichlet problem. By constructing a lower solution of a delicate form which blows up, we deduce that the solution $u(x, t)$ blows up in finite time for large enough initial conditions and for sufficiently large values of the parameter $\lambda$.

\section{Neumann Problem}

We start our study from the simplest case, when $u(x, t)$ satisfies Neumann boundary conditions, i.e. the boundary of the thermistor is thermally insulated, and so the (dimensionless) temperature $u(x, t)$ satisfies the problem

$$
\begin{gathered}
u_{t}=\Delta u+\frac{\lambda f(u)}{\left(\int_{\Omega} f(u) \mathrm{d} x\right)^{2}}, \quad x \in \Omega, t>0, \\
\frac{\partial u}{\partial \nu}=0, \quad x \in \partial \Omega, t>0, \\
u(x, 0)=u_{0}(x), \quad x \in \Omega,
\end{gathered}
$$

where $f(s)>0, f^{\prime}(s)<0$. Here $\partial / \partial \nu$ denotes the normal outward derivative to the boundary $\partial \Omega$. Throughout this work we assume that $u_{0}(x) \geqslant 0$.

In this case the associated steady-state problem

$$
\Delta w+\frac{\lambda f(w)}{\left(\int_{\Omega} f(w) \mathrm{d} x\right)^{2}}=0, \quad x \in \Omega, \quad \frac{\partial w}{\partial \nu}=0, \quad x \in \partial \Omega,
$$

does not permit any kind of solution for every $\lambda>0$. Actually, if we integrate the equation of problem (2.4) we get

$$
0=\frac{\lambda}{\int_{\Omega} f(w) \mathrm{d} x},
$$

which is a contradiction. We can prove the same result using maximum-principle arguments (Hopf's lemma). The lack of stationary solutions is an indication that timedependent solutions should be unbounded.

From the physical point of view, since the source term is positive, the system is provided with heat. On the other hand, the boundary condition $\partial u / \partial \nu=0$ prevents any heat from escaping. So, in such a situation, one expects the solution (concentration of heat) to become unbounded. This physical situation is described by the following theorem. 
Theorem 2.1. There exists $t^{*} \leqslant \infty$ such that $\|u(\cdot, t)\|_{\infty} \rightarrow \infty$ as $t \rightarrow t^{*}$. If $\int_{0}^{\infty} f(s) \mathrm{d} s<\infty$, then $u(x, t)$ blows up in finite time, i.e. $t^{*}<\infty$, while if $\int_{0}^{\infty} f(s) \mathrm{d} s=\infty$, then $t^{*}=\infty$. Moreover, blow-up is global and flat (uniform), i.e.

$$
\|u(\cdot, t)\|_{\infty} \sim u(x, t) \quad \text { as } t \rightarrow t^{*}-\text { for a.e. } x \in \Omega
$$

In order to prove this theorem we need the following auxiliary result (see also $[\mathbf{1 2}, \mathbf{1 5}]$ ).

Lemma 2.2. Let $T>0$ and let $z \in C^{1}([0, T) ; C(\bar{\Omega}))$, where $\Omega$ is a bounded domain of $\mathbb{R}^{N}$. Then for every $t \in[0, T)$ there exist points $\xi(t), \sigma(t) \in \bar{\Omega}$ with

$$
M(t):=\max _{x \in \bar{\Omega}} z(x, t)=z(\xi(t), t), \quad m(t):=\min _{x \in \bar{\Omega}} z(x, t)=z(\sigma(t), t),
$$

and the functions $M(t), m(t)$ are almost everywhere (a.e.) differentiable on $(0, T)$ with

$$
\frac{\mathrm{d} M}{\mathrm{~d} t}(t)=z_{t}(\xi(t), t) \quad \text { and } \quad \frac{\mathrm{d} m}{\mathrm{~d} t}(t)=z_{t}(\sigma(t), t) \quad \text { a.e. on }(0, T) .
$$

Proof. Let us fix $t \in[0, T)$. Since $z \in C^{1}([0, T) ; C(\bar{\Omega}))$ and $\Omega$ is bounded, there exist points $\xi(t), \sigma(t) \in \bar{\Omega}$ such that $M(t):=\max _{x \in \bar{\Omega}} z(x, t)=z(\xi(t), t)$ and $m(t):=$ $\min _{x \in \bar{\Omega}} z(x, t)=z(\sigma(t), t)$. In the following we prove that $M(t)$ satisfies relation $(2.5)$. The proof that $m(t)$ satisfies (2.5) as well follows the same steps and so we omit it.

Now let $s, t \in[0, T)$ be fixed. If $M(t) \leqslant M(s)$ we have

$$
0 \leqslant M(s)-M(t)=z(\xi(s), s)-\max _{x \in \bar{\Omega}} z(x, t) \leqslant z(\xi(s), s)-z(\xi(s), t),
$$

and so we conclude that

$$
|M(t)-M(s)| \leqslant\|z(t)-z(s)\|_{C(\bar{\Omega})}
$$

Now the mean-value theorem for functions in Banach spaces $(C(\bar{\Omega})$ in this case) yields (see [13])

$$
|M(t)-M(s)| \leqslant|t-s|_{0 \leqslant \tau \leqslant \max \{t, s\}}\left\|z_{t}(\tau)\right\|_{C(\bar{\Omega})}, \quad t, s \in[0, T) .
$$

Since $z_{t} \in C([0, T) ; C(\bar{\Omega}))$, we get that $M(t)$ is locally Lipschitz on $[0, T)$ and hence almost everywhere differentiable on $(0, T)$ in view of Rademacher's theorem (see [14]).

Let us fix $t \in(0, T)$, then since $z \in C^{1}([0, T) ; C(\bar{\Omega}))$ we have

$$
\sup _{y \in \Omega}\left|\frac{z(y, t+h)-z(y, t)}{h}-z_{t}(y, t)\right| \rightarrow 0 \quad \text { as } h \rightarrow 0 .
$$

By the definition of $M(t)$,

$$
M(t+h)=z(\xi(t+h), t+h) \geqslant z(\xi(t), t+h) .
$$

Therefore, given $h>0$, we obtain

$$
\frac{M(t+h)-M(t)}{h} \geqslant \frac{z(\xi(t), t+h)-z(\xi(t), t)}{h} .
$$


Now letting $h \rightarrow 0^{+}$and using (2.6) we get

$$
\liminf _{h \rightarrow 0^{+}} \frac{M(t+h)-M(t)}{h} \geqslant z_{t}(\xi(t), t), \quad t \in(0, T) .
$$

On the other hand,

$$
M(t-h)=z(\xi(t-h), t-h) \geqslant z(\xi(t), t-h),
$$

and so

$$
\frac{M(t)-M(t-h)}{h} \leqslant \frac{z(\xi(t), t)-z(\xi(t), t-h)}{h}, \quad h>0 .
$$

Again letting $h \rightarrow 0^{+}$and using (2.6) we obtain

$$
\limsup _{h \rightarrow 0^{-}} \frac{M(t+h)-M(t)}{h} \leqslant z_{t}(\xi(t), t), \quad t \in(0, T) .
$$

Since $M(t)$ is a.e. differentiable, relations (2.7) and (2.8) imply that

$$
\frac{\mathrm{d} M}{\mathrm{~d} t}(t)=z_{t}(\xi(t), t) \quad \text { a.e. on }(0, T) .
$$

This completes the proof.

Proof of Theorem 2.1. First we assume that $\int_{0}^{\infty} f(s) \mathrm{d} s<\infty$. Under this assumption and the positivity of $u$ (which is a consequence of the maximum principle) the functional

$$
Y(t)=\int_{\Omega} \int_{u(x, t)}^{\infty} f(\sigma) \mathrm{d} \sigma \mathrm{d} x
$$

is well defined and non-negative. Taking the derivative of $Y(t)$ with respect to $t$ and using equation (2.1) we obtain

$$
Y^{\prime}(t)=-\int_{\Omega} f(u) u_{t} \mathrm{~d} x=-\int_{\Omega} f(u) \Delta u \mathrm{~d} x-\frac{\lambda \int_{\Omega} f^{2}(u) \mathrm{d} x}{\left(\int_{\Omega} f(u) \mathrm{d} x\right)^{2}} .
$$

Using Jensen's inequality for $\phi(s)=s^{2}$ and integration by parts we have

$$
Y^{\prime}(t) \leqslant-\int_{\Omega} f(u) \Delta u \mathrm{~d} x-\frac{\lambda}{|\Omega|}=\int_{\Omega} f^{\prime}(u)|\nabla u|^{2} \mathrm{~d} x-\frac{\lambda}{|\Omega|} .
$$

Now using the monotonicity of $f(s)$ we finally get

$$
Y^{\prime}(t) \leqslant-\frac{\lambda}{|\Omega|}
$$

Hence

$$
0 \leqslant Y(t) \leqslant Y(0)-\frac{\lambda}{|\Omega|} t
$$


The latter yields that $u(x, t)$ cannot exist beyond $t^{*}$, where

$$
t^{*} \leqslant T_{u}^{*}=\frac{|\Omega| Y(0)}{\lambda}<\infty .
$$

Since the solution $u(x, t)$ of $(2.1)-(2.3)$ ceases to exist only when it becomes unbounded, we deduce that $\|u(\cdot, t)\|_{\infty} \rightarrow \infty$ as $t \rightarrow t^{*}-$ (finite-time blow-up). An immediate result of relation (2.9) is that as the $L^{1}$-norm of the initial conditions increases, the bound $T_{u}^{*}$ on the blow-up time, as is expected, decreases. Moreover, we can prove that $u(x, t)$ blows up globally and uniformly, i.e. $u(x, t) \sim\|u(\cdot, t)\|_{\infty}$ as $t \rightarrow t^{*}$ for a.e. $x \in \Omega$ (global and flat blow-up).

Using similar arguments to those in [21] we can prove that blow-up is global, i.e. $u(x, t) \rightarrow \infty$ as $t \rightarrow t^{*}$ for almost every $x \in \Omega$. Indeed, if we set $N(t)=\max _{x \in \bar{\Omega}} u(x, t)$, then, in view of Lemma 2.2, $N(t)$ satisfies

$$
\frac{\mathrm{d} N(t)}{\mathrm{d} t} \leqslant h(t) f(N(t)), \quad \text { for } h(t)=\frac{\lambda}{\left(\int_{\Omega} f(u(x, t)) \mathrm{d} x\right)^{2}},
$$

where we have also used the fact that the maximum of $u(x, t)$ is attained in $\Omega$. Since $f$ is decreasing we finally obtain

$$
\frac{\mathrm{d} N(t)}{\mathrm{d} t} \leqslant h(t) f(0)
$$

Given that $N(t) \rightarrow \infty$ as $t \rightarrow t^{*}$ we get that

$$
\int_{0}^{t} h(s) \mathrm{d} s \rightarrow \infty \quad \text { as } t \rightarrow t^{*}
$$

which yields, since $h(t)<\infty$ for $t<t^{*}$, that $h(t) \rightarrow \infty$ as $t \rightarrow t^{*}$ and so we deduce global blow-up. Now note that $n(t)=\min _{x \in \bar{\Omega}} u(x, t)$; then, in view of Lemma 2.2, we obtain

$$
\frac{\mathrm{d} n}{\mathrm{~d} t} \geqslant \frac{\lambda f(n)}{\left(\int_{\Omega} f(u) \mathrm{d} x\right)^{2}} \geqslant \frac{\lambda f(N)}{\left(\int_{\Omega} f(u) \mathrm{d} x\right)^{2}} \geqslant \frac{\mathrm{d} N}{\mathrm{~d} t}, \quad \text { a.e. in }\left(0, t^{*}\right),
$$

and finally $n(t)<N(t) \leqslant n(t)+C, C=N(0)-n(0)$, for every $t \in\left(0, t^{*}\right)$. The latter implies that blow-up is uniform. Moreover, $u(x, t) \rightarrow \infty$ for all $x \in \Omega$ as $t \rightarrow t^{*}$.

In the following we will show that the relation $\int_{0}^{\infty} f(s) \mathrm{d} s=\infty$ ensures that problem (2.1)-(2.3) has a global-in-time unbounded solution. Indeed, again using the fact that $N(t)$ satisfies

$$
\frac{\mathrm{d} N}{\mathrm{~d} t} \leqslant \frac{\lambda f(N)}{\left(\int_{\Omega} f(u) \mathrm{d} x\right)^{2}} \leqslant \frac{\lambda}{f(N)|\Omega|^{2}} \quad \text { a.e. in }(0, t),
$$

for every $t>0$, and integrating over $(0, t)$ yields

$$
\int_{N(0)}^{N(t)} f(s) \mathrm{d} s \leqslant \frac{\lambda}{|\Omega|^{2}} t,
$$


for every $t>0$. The latter implies that the solution cannot blow up. So either the solution exists for all times and it is bounded or it diverges, i.e. $N(t) \rightarrow \infty$ as $t \rightarrow \infty$. From the inequality

$$
\left(\int_{\Omega} u(x, t) \mathrm{d} x\right)_{t} \geqslant \frac{\lambda}{|\Omega| f(0)}>0,
$$

we deduce that $\lim _{t \rightarrow \infty} \int_{\Omega} u(x, t) \mathrm{d} x=\infty$. Hence $N(t)$ is unbounded.

Remark 2.3. Using the same method as above we can prove the existence of global and flat blow-up for the problem

$$
\begin{gathered}
u_{t}=\nabla \cdot(\kappa(u) \nabla(u))+\frac{\lambda f(u)}{\left(\int_{\Omega} f(u) \mathrm{d} x\right)^{p}}, \quad x \in \Omega, t>0, \\
\frac{\partial u}{\partial \nu}=0, \quad x \in \partial \Omega, t>0 \\
u(x, 0)=u_{0}(x), \quad x \in \Omega
\end{gathered}
$$

where $\kappa(u) \geqslant c>0$ and $p \geqslant 2$.

Remark 2.4. If $1<p<2$, we are also able, using the same method, to prove the occurrence of blow-up under the assumption

$$
\int_{0}^{\infty} f^{p-1}(s) \mathrm{d} s<\infty
$$

We consider the functional

$$
Y(t)=\int_{\Omega} \int_{u(x, t)}^{\infty} f^{p-1}(\sigma) \mathrm{d} \sigma \mathrm{d} x
$$

which is now well defined due to (2.13). Differentiating and applying integration by parts we obtain

$$
Y^{\prime}(t)=\int_{\Omega}(p-1) f^{p-2}(u) f^{\prime}(u)|\nabla u|^{2} \mathrm{~d} x-\frac{\lambda \int_{\Omega} f^{p}(u) \mathrm{d} x}{\left(\int_{\Omega} f(u) \mathrm{d} x\right)^{p}} \leqslant-\frac{\lambda \int_{\Omega} f^{p}(u) \mathrm{d} x}{\left(\int_{\Omega} f(u) \mathrm{d} x\right)^{p}},
$$

since $f(s)$ is decreasing. Making use of Hölder's inequality we obtain

$$
Y^{\prime}(t) \leqslant-\frac{\lambda}{|\Omega|^{p / q}}
$$

where $q=p /(p-1)$. Thus

$$
0 \leqslant Y(t) \leqslant Y(0)-\frac{\lambda}{|\Omega|^{p / q}} t
$$

and so $u(x, t)$ blows up in finite time $t^{*}$ where the upper bound on the blow-up time is now given by the relation

$$
T_{u}^{*}=\frac{Y(0)|\Omega|^{p / q}}{\lambda} .
$$

Again we can prove that blow-up is global and uniform. 


\section{Robin problem}

Now we discuss the problem applying a Robin condition, i.e. considering Newtonian cooling, over the whole boundary $\partial \Omega$ :

$$
\begin{gathered}
u_{t}=\Delta u+\frac{\lambda f(u)}{\left(\int_{\Omega} f(u) \mathrm{d} x\right)^{2}}, \quad x \in \Omega, t>0, \\
\frac{\partial u}{\partial \nu}+\beta u(x, t)=0, \quad x \in \partial \Omega, t>0, \\
u(x, 0)=u_{0}(x), \quad x \in \Omega,
\end{gathered}
$$

where $\beta=\beta(x)>0$. We still assume that $f(s)>0, f^{\prime}(s)<0$ and $\int_{0}^{\infty} f(s) \mathrm{d} s<\infty$; actually, for simplicity we take

$$
\int_{0}^{\infty} f(s) \mathrm{d} s=1 .
$$

The main result of this section is the following theorem.

Theorem 3.1. If $u(x, t)$ is a solution of (3.1)-(3.3), then for $\lambda$ sufficiently large there exists $t^{*}<\infty$ such that $\|u(\cdot, t)\|_{\infty} \rightarrow \infty$ as $t \rightarrow t^{*}-$, i.e. $u(x, t)$ blows up in finite time. Moreover, blow-up is global and flat.

Proof. In this case the technique used for the Neumann problem fails because when integrating by parts the term containing $\partial u / \partial \nu$ does not vanish any more on the boundary. In order to overcome this difficulty we have to properly modify the form of the functional $Y(t)$, so that the boundary terms are killed. This can be done using an auxiliary function in the definition of $Y(t)$. More precisely, we now consider the functional

$$
Y(t)=\int_{\Omega} \Psi(x)\left(\int_{u(x, t)}^{\infty} f(\sigma) \mathrm{d} \sigma\right) \mathrm{d} x
$$

where $\Psi(x)$ is the Robin eigenfunction corresponding to the principal eigenvalue of the Laplacian, i.e. $\Psi(x)$ satisfies the problem

$$
-\Delta \Psi=\mu_{1} \Psi, \quad x \in \Omega, \quad \frac{\partial \Psi}{\partial \nu}+\beta \Psi=0, \quad x \in \partial \Omega .
$$

It is known (see, for example, $\left[\mathbf{2}\right.$, Theorem 4.3]) that $\mu_{1}$ is positive and that $\Psi(x)$ does not change sign in $\bar{\Omega}$, so it can be chosen to be positive and for simplicity normalized so that

$$
\int_{\Omega} \Psi(x) \mathrm{d} x=1
$$

Hence the functional $Y(t)$ is non-negative and well defined because of the relation (3.4).

Differentiating $Y(t)$ and using (3.1) we obtain

$$
Y^{\prime}(t)=-\int_{\Omega} \Psi(x) f(u) u_{t}=-\int_{\Omega} \Psi(x) f(u) \Delta u \mathrm{~d} x-\frac{\lambda \int_{\Omega} f^{2}(u) \Psi(x) \mathrm{d} x}{\left(\int_{\Omega} f(u) \mathrm{d} x\right)^{2}} .
$$


Integrating by parts and using Jensen's inequality we obtain

$$
\begin{aligned}
Y^{\prime}(t) & \leqslant-\int_{\partial \Omega} \Psi(s) f(u) \frac{\partial u}{\partial \nu} \mathrm{d} s+\int_{\Omega} \nabla(\Psi(x) f(u)) \cdot \nabla u \mathrm{~d} x-\frac{\lambda m}{|\Omega|} \\
& =\int_{\partial \Omega} \beta(s) \Psi(s) f(u) u \mathrm{~d} s+\int_{\Omega} \nabla(\Psi(x)) f(u) \cdot \nabla u \mathrm{~d} x+\int_{\Omega} \Psi(x) f^{\prime}(u)|\nabla u|^{2} \mathrm{~d} x-\frac{\lambda m}{|\Omega|},
\end{aligned}
$$

where $m=\min _{x \in \bar{\Omega}} \Psi(x)>0$.

Using the monotonicity of $f$ we have

$$
\begin{aligned}
Y^{\prime}(t) & \leqslant \int_{\partial \Omega} \beta \Psi f(u) u \mathrm{~d} s+\int_{\partial \Omega} \frac{\partial \Psi}{\partial \nu}\left(\int_{0}^{u} f(\sigma) \mathrm{d} \sigma\right) \mathrm{d} s-\int_{\Omega} \Delta \Psi\left(\int_{0}^{u} f(\sigma) \mathrm{d} \sigma\right) \mathrm{d} x-\frac{\lambda m}{|\Omega|} \\
& =\int_{\partial \Omega} \beta \Psi f(u) u \mathrm{~d} s-\int_{\partial \Omega} \beta \Psi\left(\int_{0}^{u} f(\sigma) \mathrm{d} \sigma\right) \mathrm{d} s+\mu_{1} \int_{\Omega} \Psi\left(\int_{0}^{u} f(\sigma) \mathrm{d} \sigma\right) \mathrm{d} x-\frac{\lambda m}{|\Omega|} \\
& \leqslant \mu_{1} \int_{\Omega} \Psi\left(\int_{0}^{\infty} f(\sigma) \mathrm{d} \sigma\right) \mathrm{d} x-\frac{\lambda m}{|\Omega|} \\
& =\mu_{1}-\frac{\lambda m}{|\Omega|}
\end{aligned}
$$

where relations (3.4) and (3.6) have also been taken into account. If we integrate the last relation over $(0, t)$ we get

$$
Y(t) \leqslant Y(0)-\left(\frac{\lambda m}{|\Omega|}-\mu_{1}\right) t
$$

and so if we choose $\lambda>\hat{\lambda}=\mu_{1}|\Omega| / m$, we observe that $u(x, t)$ cannot be extended beyond

$$
T_{u}^{*}=\frac{Y(0)}{(\lambda m /|\Omega|)-\mu_{1}},
$$

and so it should blow up in finite time $t^{*} \leqslant T_{u}^{*}$. Using the same arguments as in the analysis of the Neumann problem it can be proven that blow-up is also global. The uniform nature of blow-up can be proven either by using arguments similar to those used in the Neumann problem or by using the same arguments used for the Dirichlet problem (see the next section).

For the Robin problem, our method, apart from the occurrence of blow-up, provides us with an upper estimate of the critical parameter, say $\lambda^{*}$, above which the corresponding steady-state problem

$$
\Delta w+\frac{\lambda f(w)}{\left(\int_{\Omega} f(w) \mathrm{d} x\right)^{2}}=0, \quad x \in \Omega, \quad \frac{\partial w}{\partial \nu}+\beta w(x)=0, \quad x \in \partial \Omega,
$$

has no solutions. Indeed, we claim that $\lambda^{*} \leqslant \hat{\lambda}$, because otherwise, for initial conditions $u(x, 0)<w_{m}(x ; \lambda)$, where $w_{m}(x ; \lambda)$ is the minimal solution of problem (3.7) corresponding to $\hat{\lambda}<\lambda<\lambda^{*}$, we could prove, using ideas similar to those in [21], that $u(x, t) \rightarrow w_{m}(x)$ as $t \rightarrow \infty$, which is a contradiction since blow-up occurs for every initial 
condition if $\lambda>\hat{\lambda}$. This estimate seems not to be optimal, at least for $f(s)=\mathrm{e}^{-s}$, since Bebernes and Lacey conjectured (in $[\mathbf{6}]$ ) that $\lambda^{*}$, in this case, should be proportional to $2|\partial \Omega|^{2}$. However, for the one-dimensional case our estimate $\hat{\lambda}$ improves the existing ones in the literature (see $[\mathbf{2 1}, \mathbf{2 3}]$ ).

Remark 3.2. In the case of a temperature-dependent thermal conductivity $\kappa(u)>0$, $u$ satisfies the problem

$$
\begin{gathered}
u_{t}=\nabla(\kappa(u) \nabla u)+\frac{\lambda f(u)}{\left(\int_{\Omega} f(u) \mathrm{d} x\right)^{2}}, \quad x \in \Omega, t>0, \\
\frac{\partial u}{\partial \nu}+\beta u(x, t)=0, \quad x \in \partial \Omega, t>0, \\
u(x, 0)=u_{0}(x), \quad x \in \Omega,
\end{gathered}
$$

we can prove that blow-up occurs under the additional assumption that $\kappa(s)$ satisfies the conditions $(f(s) \kappa(s))^{\prime}<0, s>0$ and $\int_{0}^{\infty} f(s) \kappa(s) \mathrm{d} s<\infty$, or

$$
\int_{0}^{\infty} f(s) \kappa(s) \mathrm{d} s=1,
$$

after scaling. Indeed, considering again the functional $Y(t)$, differentiating with respect to $t$ and using equation (3.8) we obtain

$$
Y^{\prime}(t) \leqslant-\int_{\Omega} \Psi(x) f(u) \nabla(\kappa(u) \nabla u) \mathrm{d} x-\frac{\lambda m}{|\Omega|},
$$

where we have also applied Jensen's inequality; we recall that $m=\min _{x \in \bar{\Omega}} \Psi(x)>0$ and $\Psi(x)>0$ is the eigenfunction associated with the principal eigenvalue $\mu_{1}$ of problem (3.5) satisfying (3.6).

Using integration by parts now, we get

$$
\begin{aligned}
Y^{\prime}(t) \leqslant & -\int_{\partial \Omega} \Psi f(u) \kappa(u) \frac{\partial u}{\partial \nu} \mathrm{d} s+\int_{\Omega} \nabla(\Psi(x) f(u)) \kappa(u) \cdot \nabla(u) \mathrm{d} x-\frac{\lambda m}{|\Omega|} \\
= & \beta \int_{\partial \Omega} \Psi f(u) \kappa(u) u \mathrm{~d} s+\int_{\Omega} \nabla \Psi(x) \cdot \nabla\left(\int_{0}^{u} f(\sigma) \kappa(\sigma) \mathrm{d} \sigma\right) \mathrm{d} x \\
& \quad+\int_{\Omega} \Psi(x) f^{\prime}(u) \kappa(u)|\nabla u|^{2} \mathrm{~d} x-\frac{\lambda m}{|\Omega|} \quad \text { (due to the boundary conditions) } \\
\leqslant & \beta \int_{\partial \Omega} \Psi f(u) \kappa(u) u \mathrm{~d} s-\beta \int_{\partial \Omega} \Psi\left(\int_{0}^{u} f(\sigma) \kappa(\sigma) \mathrm{d} \sigma\right) \mathrm{d} s \\
& \left.\quad-\int_{\Omega} \Delta \Psi\left(\int_{0}^{u} f(\sigma) \kappa(\sigma) \mathrm{d} \sigma\right) \mathrm{d} x-\frac{\lambda m}{|\Omega|} \quad \text { (since } f^{\prime}(s)<0\right) \\
= & \mu_{1}-\frac{\lambda m}{|\Omega|} \quad\left(\text { since }(f(s) \kappa(s))^{\prime}<0 \text { and }(3.6),(3.11)\right. \text { are full field). }
\end{aligned}
$$

The latter yields

$$
0<Y(t) \leqslant Y(0)-\left(\frac{\lambda m}{|\Omega|}-\mu_{1}\right) t
$$


and finally we conclude that the solution of (3.8)-(3.10) blows up globally in finite time by

$$
T_{u}^{*}=\frac{Y(0)}{(\lambda m /|\Omega|)-\mu_{1}},
$$

provided that $\lambda>\hat{\lambda}=\mu_{1}|\Omega| / m$. It can also be proven that blow-up is global and uniform.

When the exponent of the non-local term is $p>2$ we can prove again that the solution of the corresponding time-dependent problem blows up for large values of the parameter $\lambda$. We consider again $Y(t)$ and following the same steps as in the case $p=2$ we obtain

$$
Y^{\prime}(t) \leqslant \mu_{1}-\lambda m \frac{\int_{\Omega} f^{2}(u) \mathrm{d} x}{\left(\int_{\Omega} f(u) \mathrm{d} x\right)^{p}} .
$$

Using Jensen's inequality, the above relation takes the form

$$
Y^{\prime}(t) \leqslant \mu_{1}-\frac{\lambda m}{|\Omega|^{p-1} f^{p-2}(0)},
$$

where we have also used the fact that $f(s)$ is decreasing. Thus

$$
0 \leqslant Y(t) \leqslant Y(0)-\left(\frac{\lambda m}{|\Omega|^{p-1} f^{p-2}(0)}-\mu_{1}\right) t
$$

and if we choose

$$
\lambda>\hat{\lambda}=\frac{\mu_{1}|\Omega|^{p-1} f^{p-2}(0)}{m},
$$

then we finally observe that $u(x, t)$ blows up, again uniformly and globally, as $t \rightarrow t^{*}$, where

$$
t^{*} \leqslant T_{u}^{*}=\frac{Y(0)}{\left(\lambda m /|\Omega|^{p-1} f^{p-2}(0)\right)-\mu_{1}} .
$$

It can also be proven that $\hat{\lambda}$ is an upper bound of critical value $\lambda^{*}$.

Remark 3.3. For $1<p<2$, we should again impose the condition that $f(s)$ satisfies $\int_{0}^{\infty} f^{p-1}(s) \mathrm{d} s<\infty$ or, after scaling,

$$
\int_{0}^{\infty} f^{p-1}(s) \mathrm{d} s=1 .
$$

It is enough to consider the functional

$$
Y(t)=\int_{\Omega} \Psi(x)\left(\int_{u(x, t)}^{\infty} f^{p-1}(\sigma) \mathrm{d} \sigma\right) \mathrm{d} x
$$

which is well defined and positive; here $\Psi(x)$ is again the principal eigenfunction of problem (3.5) normalized by the condition (3.6). Following the same steps as in the case $p=2$ and using the monotonicity of $f^{p-1}(s)$, we get that

$$
Y^{\prime}(t) \leqslant \mu_{1}-\frac{\lambda m \int_{\Omega} f^{p}(u) \mathrm{d} x}{\left(\int_{\Omega} f(u) \mathrm{d} x\right)^{p}} .
$$


This last relation takes the form

$$
Y^{\prime}(t) \leqslant \mu_{1}-\frac{\lambda m}{|\Omega|^{p / q}},
$$

in view of Hölder's inequality, where $q=p /(p-1)$, and so we obtain that

$$
0 \leqslant Y(t) \leqslant Y(0)-\left(\frac{\lambda m}{|\Omega|^{p / q}}-\mu_{1}\right) t .
$$

Consequently, for $\lambda>\hat{\lambda}=\mu_{1}|\Omega|^{p / q} / m$, we deduce that $\|u(\cdot, t)\|_{\infty} \rightarrow \infty$ as $t \rightarrow t^{*}$, where

$$
t^{*} \leqslant T_{u}^{*}=\frac{Y(0)}{\left(\lambda m /|\Omega|^{p / q}\right)-\mu_{1}} .
$$

Apart from the fact that blow-up is global, we can also prove that it is uniform as well. Moreover, $\hat{\lambda}$ again provides an upper-bound estimate of the critical value $\lambda^{*}$.

\section{The Dirichlet problem}

In this section we deal with the problem

$$
\begin{gathered}
u_{t}=\Delta u+\frac{\lambda f(u)}{\left(\int_{\Omega} f(u) \mathrm{d} x\right)^{2}}, \quad x \in \Omega, t>0, \\
u(x, t)=0, \quad x \in \partial \Omega, t>0 \\
u(x, 0)=u_{0}(x), \quad x \in \Omega .
\end{gathered}
$$

In this case we expect blow-up to occur if $f(s)$ satisfies the condition

$$
\int_{0}^{\infty} f(s) \mathrm{d} s<\infty
$$

Indeed, under (4.4) there exists a critical parameter $\lambda^{*} \geqslant 2|\partial \Omega|^{2}$ (see Theorem 2.2 in $[6]$ ) such that the steady-state problem

$$
\Delta w+\frac{\lambda f(w)}{\left(\int_{\Omega} f(w) \mathrm{d} x\right)^{2}}=0, \quad x \in \Omega, w(x)=0, x \in \partial \Omega
$$

has no solutions for $\lambda>\lambda^{*}$.

If we try to prove blow-up again using the (positive) functional

$$
Y(t)=\int_{\Omega} \Psi(x)\left(\int_{u(x, t)}^{\infty} f(\sigma) \mathrm{d} \sigma\right) \mathrm{d} x,
$$

where now $\Psi(x)>0$ is the eigenfunction corresponding to the principal eigenvalue $\mu_{1}$ of $-\Delta$ with Dirichlet conditions, we obtain that

$$
Y^{\prime}(t) \leqslant \mu_{1}-\frac{\lambda \int_{\Omega} f^{2}(u) \Psi(x) \mathrm{d} x}{\left(\int_{\Omega} f(u) \mathrm{d} x\right)^{2}} .
$$


But this relation does not enable us, as it does in the Robin case, to deduce blow-up because now it seems difficult to find a positive lower (time-independent) bound for the quantity

$$
\frac{\lambda \int_{\Omega} f^{2}(u) \Psi(x) \mathrm{d} x}{\left(\int_{\Omega} f(u) \mathrm{d} x\right)^{2}} .
$$

Thus we will prove blow-up, for $\lambda>\lambda^{*}$, by constructing a lower solution of problem (4.1)-(4.3) that blows up. Our main result in this section is the following theorem.

Theorem 4.1. For $\lambda>\lambda^{*}$ and sufficiently large initial conditions the solution of the problem (4.1)-(4.3) blows up globally and uniformly in finite time provided that $f(s)$ satisfies $\int_{0}^{\infty}\left[s f(s)-s^{2} f^{\prime}(s)\right] \mathrm{d} s<\infty$.

Proof. For the construction of this lower solution we will need to first define a lower solution of problem (4.5), which was first introduced by Bebernes and Lacey in [6]. This lower solution has the form

$$
v(x ; \mu, \nu, \delta)= \begin{cases}V(\sqrt{\nu \delta} d(x, \partial \Omega) ; \mu), & d(x, \partial \Omega)<\frac{\mu}{\sqrt{\nu \delta}}, \\ V(\mu ; \mu)=M, & d(x, \partial \Omega) \geqslant \frac{\mu}{\sqrt{\nu \delta}}\end{cases}
$$

where $V$ is the solution of the problem

$$
V^{\prime \prime}+f(V)=0, \quad 0<y<\mu, \quad V(0 ; \mu)=0, \quad V^{\prime}(\mu ; \mu)=0,
$$

while $\delta=\lambda /\left(\int_{\Omega} f(w) \mathrm{d} x\right)^{2}$ is the so-called local parameter of (4.5) and $\nu$ is a constant to be determined later. Here $d(x, \partial \Omega)$ stands for the distance between $x \in \Omega$ and the boundary $\partial \Omega$. As is indicated in $[6], d$ is smooth and more precisely $|\Delta d|<K$, for some $K$, in a neighbourhood of the boundary if $\partial \Omega$ is smooth. In particular, such a neighbourhood consists of all $x \in \Omega$ such that $d(x, \partial \Omega) \leqslant \mu / \sqrt{\nu \delta}<\rho$ where $\rho$ is smaller than the infimum of the radius of the largest interior ball touching the boundary at some $z \in \partial \Omega$. Hence in the following $\delta$ is chosen large enough to ensure that $\mu / \sqrt{\nu \delta}<\rho$.

Now we proceed as in $[6]$. Obviously, $v$ satisfies the correct boundary condition and is $C^{1}$ while

$$
\begin{aligned}
\Delta v+\delta f(v) & =\delta f(V(\mu))>0 \quad \text { for } d(x, \partial \Omega) \geqslant \frac{\mu}{\sqrt{\nu \delta}} \\
\Delta v+\delta f(v) & =\nu \delta|\nabla d|^{2} V^{\prime \prime}+\sqrt{\nu \delta} V^{\prime} \Delta d+\delta f(V) \\
& =(1-\nu) \delta f(V)+\sqrt{\nu \delta} V^{\prime} \Delta d \quad \text { where } d(x, \partial \Omega)<\frac{\mu}{\sqrt{\nu \delta}} \\
& >(1-\nu) \delta f(V)-\sqrt{\delta} K V^{\prime} \geqslant 0 \quad \text { for } \nu<1,
\end{aligned}
$$

provided that $\nu \leqslant 1-(K G(\mu) / \sqrt{\delta})$, where $G(\mu)=\sup _{y \in(0, \mu)}\left(V^{\prime} / f(V)\right)$. So, on choosing $\nu=1-K G(\mu) / \sqrt{\delta}, v$ is a lower solution of problem (4.5) for sufficiently large $\delta$, i.e. for $\lambda<\lambda^{*}$ sufficiently close to $\lambda^{*}$. 
Problem (4.7) yields that $V$ satisfies

$$
V^{\prime 2}=2 \int_{V}^{M} f(s) \mathrm{d} s=2 \int_{V}^{\infty} f(s) \mathrm{d} s-2 \int_{M}^{\infty} f(s) \mathrm{d} s=2(F(V)-F(M)),
$$

where $F(\sigma)=\int_{\sigma}^{\infty} f(s) \mathrm{d} s$ and so the relation between $\mu$ and $M$ is defined by

$$
\mu=\mu(M)=\frac{1}{\sqrt{2}} \int_{0}^{M}[F(s)-F(M)]^{-1 / 2} \mathrm{~d} s
$$

We now consider as a candidate lower solution the function

$$
z(x, t)= \begin{cases}V(\sqrt{\nu \delta} d(x, \partial \Omega) ; \mu), & d(x, \partial \Omega)<\frac{\mu}{\sqrt{\nu \delta}} \\ V(\mu ; \mu)=M(t), & d(x, \partial \Omega) \geqslant \frac{\mu}{\sqrt{\nu \delta}}\end{cases}
$$

where the functions $\mu=\mu(t), M=M(t), \delta=\delta(t)$ and the constant $\nu$ should be determined. Actually, due to (4.8) the dependence between $M(t)$ and $\mu(t)$ is determined, while we are free to choose the dependence between $M(t)$ and $\delta(t)$. It is evident, from the definition of $z(x, t)$ that, if the ratio $\mu / \sqrt{\nu \delta}$ decreases to 0 as $t$ increases, then the spatial independent behaviour of $z(x, t)$ dominates the behaviour near the boundary, i.e. the growth of $z(x, t)$ is uniform (flat) as $t$ increases. In the following we will choose $M(t)$ and the dependence between $M$ and $\delta$ such that, for large enough initial conditions, $z(x, t)$ is a lower solution of problem (4.1)-(4.3) which blows up in finite time. Indeed, we choose

$$
\delta=\delta(M)=M^{2} / f(M),
$$

while we impose the condition that $M(t)$ satisfies

$$
\dot{M}(t):=\frac{\mathrm{d} M}{\mathrm{~d} t}=\frac{\lambda-\lambda^{*}}{\left(\int_{\Omega} f(z) \mathrm{d} x\right)^{2}} \inf _{x \in \Omega}\left\{\frac{f(z)}{z_{M}}\right\}>0 \text { for } t>0
$$

and $M(0)$ is chosen large enough so that $\delta_{0}=\delta(M(0))=M^{2}(0) / f(M(0))$ is also sufficiently large (note that $\delta^{\prime}(M)>0$ ). We have

$$
z_{M}=\frac{\partial z}{\partial M}=\frac{\partial V}{\partial y} y^{\prime}(M)+\frac{\partial V}{\partial \mu} \mu^{\prime}(M)>0
$$

where $y=y(M)=\sqrt{\nu \delta(M)} d(x, \partial \Omega)$. In fact, differentiating problem (4.7) with respect to $y$ and using the monotonicity of $f(s)$ together with maximum-principle arguments we get $\partial V / \partial y>0$. Besides, $y^{\prime}(M)=\frac{1}{2} y(M) \delta^{\prime}(M) / \delta(M)>0$ and $\mu^{\prime}(M), V_{\mu}(y ; \mu)>0$. After scaling, $Y=y / \mu$ yields that problem (4.7) can be written in the form

$$
W^{\prime \prime}+\mu^{2} f(W)=0, \quad 0<Y<1, \quad W(0 ; \mu)=0, \quad W^{\prime}(1 ; \mu)=0,
$$

where $W(Y)=V(y)$. Differentiating this problem with respect to $\mu$ we obtain

$$
-W_{\mu}^{\prime \prime}-\mu^{2} f^{\prime}(W) W_{\mu}=2 \mu f(W)>0, \quad 0<Y<1, \quad W_{\mu}(0 ; \mu)=0, \quad W_{\mu}^{\prime}(1 ; \mu)=0,
$$


which implies, via the maximum principle, that $W_{\mu}(Y ; \mu)=V_{\mu}(y ; \mu)>0$ for $0<y<\mu$ and $W_{\mu}(1 ; \mu)=M^{\prime}(\mu)>0$, hence $\mu^{\prime}(M)>0$.

Now if we choose $\delta_{0} \gg 1$, then since $\delta^{\prime}(M)>0$ and $M(t)$ satisfies (4.10) we obtain that $\delta(t) \gg 1$ for every $t>0$. Hence

$$
-\Delta z \leqslant \delta(t) f(z) \text { for every } t>0
$$

provided that $\nu$ is chosen such that

$$
\nu \leqslant \inf _{M>M(0)}\left(1-\frac{K G(M)}{\sqrt{\delta(M)}}\right)
$$

and $M(0) \gg 1$ (see also the comments after the definition of $v(x ; \mu, \nu, \delta)$ ). This choice is possible since $G(M) / \sqrt{\delta(M)} \rightarrow 0$ as $M \rightarrow \infty$ (see below).

Thus

$$
\begin{aligned}
z_{t}-\Delta z-\frac{\lambda f(z)}{\left(\int_{\Omega} f(z) \mathrm{d} x\right)^{2}} & =z_{M} \dot{M}(t)-\Delta z-\frac{\lambda f(z)}{\left(\int_{\Omega} f(z) \mathrm{d} x\right)^{2}} \\
& \leqslant z_{M} \dot{M}(t)+\delta(t) f(z)-\frac{\lambda f(z)}{\left(\int_{\Omega} f(z) \mathrm{d} x\right)^{2}} \quad \text { due to (4.11) } \\
& \lesssim z_{M} \dot{M}(t)+\frac{\left[\lambda^{*}-\lambda\right] f(z)}{\left(\int_{\Omega} f(z) \mathrm{d} x\right)^{2}} \\
& =z_{M}\left(\frac{\lambda-\lambda^{*}}{\left(\int_{\Omega} f(z) \mathrm{d} x\right)^{2}} \inf _{x \in \Omega}\left\{\frac{f(z)}{z_{M}}\right\}+\frac{\left[\lambda^{*}-\lambda\right]}{\left(\int_{\Omega} f(z) \mathrm{d} x\right)^{2}} \frac{f(z)}{z_{M}}\right) \\
& =\frac{z_{M}\left(\lambda-\lambda^{*}\right)}{\left(\int_{\Omega} f(z) \mathrm{d} x\right)^{2}}\left[\inf _{x \in \Omega}\left\{\frac{f(z)}{z_{M}}\right\}-\frac{f(z)}{z_{M}}\right] \leqslant 0,
\end{aligned}
$$

since $\delta(t) \lesssim \lambda^{*} /\left(\int_{\Omega} f(z) \mathrm{d} x\right)^{2}$ holds for $M(0)$ sufficiently large, which results from $(*)$, (4.18) and the definition of $z(x, t)$. Hence, if $M(t)$ satisfies (4.10), then $z(x, t)$ is a lower solution of problem (4.1)-(4.3) for sufficiently large initial conditions. In the following we show that (4.10) also permits us to deduce blow-up for the lower solution $z(x, t)$.

First we notice that

$$
\inf _{x \in \Omega}\left\{\frac{f(z)}{z_{M}}\right\}=\min \left\{f(M), \inf _{d(x, \partial \Omega)<\mu / \sqrt{\nu \delta}}\left\{\frac{f(z)}{z_{M}}\right\}\right\},
$$

where

$$
\inf _{d(x, \partial \Omega)<\mu / \sqrt{\nu \delta}}\left\{\frac{f(z)}{z_{M}}\right\}=\inf _{y \in(0, \mu)}\left\{\frac{f(V(y ; \mu))}{V_{M}(y ; \mu)}\right\}=\frac{1}{\sup _{y \in(0, \mu)}\left\{V_{M}(y ; \mu) / f(V(y ; \mu))\right\}} .
$$

Since

$$
V_{M}(y ; \mu)=\frac{1}{2} y \frac{\delta^{\prime}(M)}{\delta(M)} \frac{\partial V}{\partial y}+\frac{\partial V}{\partial \mu} \mu^{\prime}(M)
$$


then

$$
\begin{aligned}
\sup _{y \in(0, \mu)}\left\{\frac{V_{M}(y ; \mu)}{f(V(y ; \mu))}\right\} & \leqslant \frac{1}{2} \frac{\delta^{\prime}(M)}{\delta(M)} \sup _{y \in(0, \mu)}\left\{\frac{V_{y}(y ; \mu) y}{f(V(y ; \mu))}\right\}+\sup _{y \in(0, \mu)}\left\{\frac{V_{\mu}(y ; \mu) \mu^{\prime}(M)}{f(V(y ; \mu))}\right\} \\
& \leqslant \frac{1}{2} \frac{\delta^{\prime}(M)}{\delta(M)} G(\mu(M)) \mu(M)+\sup _{y \in(0, \mu)}\left\{\frac{V_{\mu}(y ; \mu)}{f(V(y ; \mu))}\right\} \mu^{\prime}(M) .
\end{aligned}
$$

But

$$
\mu(M) / \sqrt{\delta(M)} \ll 1 \quad \text { as } M \rightarrow \infty
$$

and

$$
G(\mu(M)) / \sqrt{\delta(M)} \ll 1 \quad \text { as } M \rightarrow \infty
$$

hold. The former follows from the relation (4.8):

$$
\begin{aligned}
\mu(M) & =\frac{1}{\sqrt{2}} \int_{0}^{M}[F(s)-F(M)]^{-1 / 2} \mathrm{~d} s \\
& \leqslant \frac{1}{\sqrt{2}} \int_{0}^{M}[(M-s) f(M)]^{-1 / 2} \mathrm{~d} s \\
& =\sqrt{\frac{2 M}{f(M)}} \ll \frac{M}{\sqrt{f(M)}}=\sqrt{\delta(M)} \quad \text { as } M \rightarrow \infty .
\end{aligned}
$$

Now, taking into account the monotonicity of $f$ we notice that

$$
f^{2}(M)(F(V)-F(M)) / f^{2}(V) \leqslant(M-V) f^{2}(M) / f(V) \leqslant(M-V) f(M) \leqslant M f(M),
$$

which implies

$$
\begin{aligned}
G(\mu(M)) & =\sup _{V \in(0, M)} \frac{\sqrt{2(F(V)-F(M))}}{f(V)} \\
& \leqslant \sqrt{\frac{2 M}{f(M)}} \\
& \ll \frac{M}{\sqrt{f(M)}} \\
& =\sqrt{\delta(M)} \text { as } M \rightarrow \infty .
\end{aligned}
$$

Moreover,

$$
\sup _{y \in(0, \mu)}\left\{\frac{V_{\mu}(y ; \mu)}{f(V(y ; \mu))}\right\} \leqslant \frac{V_{\mu}(\mu ; \mu)}{f(V(\mu ; \mu))}=\frac{M^{\prime}(\mu)}{f(M)}
$$

since

$$
\frac{\partial^{2} V(y ; \mu)}{\partial \mu \partial y}=-\int_{0}^{y} f^{\prime}(V(s ; \mu)) V_{\mu}(s ; \mu) \mathrm{d} s+\frac{\partial^{2} V(0 ; \mu)}{\partial \mu \partial y} \geqslant 0 .
$$


Now relation (4.13) using (4.14), (4.15) and (4.16) takes the form

$$
\sup _{y \in(0, \mu)}\left\{\frac{V_{M}(y)}{f(V(y))}\right\}<\frac{1}{2} \delta^{\prime}(M)+\frac{1}{f(M)} \sim \frac{1}{2} \delta^{\prime}(M) \quad \text { for } M \gg 1,
$$

since

$$
\lim _{M \rightarrow \infty} \frac{2 / \delta^{\prime}(M)}{f(M)}=\lim _{M \rightarrow \infty} \frac{2}{2 M-\left(M^{2} f^{\prime}(M) / f(M)\right)}=0 .
$$

Thus

$$
\inf _{x \in \Omega}\left\{\frac{f(z)}{z_{M}}\right\} \gtrsim \min \left\{f(M), \frac{2}{\delta^{\prime}(M)}\right\} \sim \frac{2}{\delta^{\prime}(M)} \quad \text { for } M \gg 1 .
$$

Consequently, in view of relation (4.10) we obtain

$$
\begin{aligned}
\dot{M}(t) \gtrsim \frac{\lambda-\lambda^{*}}{\left(\int_{\Omega} f(z) \mathrm{d} x\right)^{2}} \frac{2}{\delta^{\prime}(M)} & \sim \frac{2\left(\lambda-\lambda^{*}\right) / \delta^{\prime}(M)}{|\Omega|^{2} f^{2}(M)} \\
& =\frac{\left(\lambda-\lambda^{*}\right)}{|\Omega|^{2}\left[2 M f(M)-M^{2} f^{\prime}(M)\right]}, \quad M \gg 1,
\end{aligned}
$$

since $\int_{\Omega} f(z) \mathrm{d} x \sim f(M)|\Omega|$ as $M \rightarrow \infty$ due to (4.14) and the definition of $z(x, t)$. The latter implies that $M(t) \rightarrow \infty$ as $t \rightarrow t_{1}^{*}$, where

$$
t_{1}^{*} \lesssim \frac{|\Omega|^{2}}{\left(\lambda-\lambda^{*}\right)} \int_{M(0)}^{\infty}\left[2 \sigma f(\sigma)-\sigma^{2} f^{\prime}(\sigma)\right] \mathrm{d} \sigma<\infty
$$

provided that $f$ also satisfies the condition

$$
\int_{M(0)}^{\infty}\left[2 \sigma f(\sigma)-\sigma^{2} f^{\prime}(\sigma)\right] \mathrm{d} \sigma<\infty
$$

i.e. the lower solution $z(x, t)$ blows up in finite time $t_{1}^{*}$, provided that $M(0) \gg 1$.

Thus the solution $u(x, t)$ of problem (4.1)-(4.3) blows up in finite time $t^{*} \leqslant t_{1}^{*}$, i.e. $\|u(\cdot, t)\|_{\infty} \rightarrow \infty$ as $t \rightarrow t^{*}$, for large enough initial conditions. It can be proven, using the same arguments as in the Neumann problem, that blow-up is global. Moreover, the upper bound of blow-up time given by relation (4.17) is of the same form with the upper estimate obtained in [18] for the Robin problem and in the one-dimensional case. We can prove, using the same arguments as in [20], that blow-up is also uniform (flat) in this case. Actually, if we consider the problem

$$
\begin{aligned}
v_{t} & =\Delta v+h(t) f(N), \quad x \in \Omega, t>0, \\
v(x, t) & =0, \quad x \in \partial \Omega, t>0, \\
v(x, 0) & =0, \quad x \in \Omega .
\end{aligned}
$$

where $N=N(t)=\max _{x \in \Omega} u(x, t)$ and $h(t)=\lambda /\left(\int_{\Omega} f(u(x, t)) \mathrm{d} x\right)^{2}$, then $v(x, t)$ is a lower solution of problem (4.1)-(4.3) since $f(s)$ is decreasing. We can write the solution of the above problem as $v(x, t)=\theta(x, t)+V(t)$, where $V(t)$ is the solution of the problem

$$
\frac{\mathrm{d} V(t)}{\mathrm{d} t}=h(t) f(N), \quad V(0)=0
$$


whereas $\theta(x, t)$ should satisfy

$$
\begin{aligned}
\theta_{t} & =\Delta \theta, & & x \in \Omega, t>0, \\
\theta(x, t) & =-V(t), & & x \in \partial \Omega, t>0, \\
\theta(x, 0) & =0, & & x \in \Omega,
\end{aligned}
$$

and so has the integral representation

$$
\theta(x, t)=\int_{0}^{t} V(\tau) \int_{\partial \Omega} \frac{\partial G(x, s, t-\tau)}{\partial \nu} \mathrm{d} s \mathrm{~d} \tau .
$$

Here $G(x, y, t)$ is the Green function for the heat equation in $\Omega$ with Dirichlet boundary conditions. Thus

$$
v(x, t)=V(t)+\int_{0}^{t} V(\tau) \int_{\partial \Omega} \frac{\partial G(x, s, t-\tau)}{\partial \nu} \mathrm{d} s \mathrm{~d} \tau,
$$

and for any fixed $x \in \Omega$ the second term on the right-hand side of (4.19) is much smaller than the first as $t \rightarrow t^{*}$, due to the contribution of the Green function term, and so $v(x, t) \sim V(t)$ as $t \rightarrow t^{*}$ for $x \in \Omega$. Hence $N(t) \geqslant u(x, t) \gtrsim V(t)$ as $t \rightarrow t^{*}$ for $x \in \Omega$ and, since

$$
\frac{\mathrm{d} N(t)}{\mathrm{d} t} \leqslant h(t) f(N)=\frac{\mathrm{d} V(t)}{\mathrm{d} t},
$$

we obtain $N(t) \lesssim V(t) \lesssim N(t)$ as $t \rightarrow t^{*}$. So it follows that $V(t) \sim N(t)$, and consequently we deduce $u(x, t) \sim N(t)$ as $t \rightarrow t^{*}-$ for every $x \in \Omega$ (uniform blow-up).

Remark 4.2. Relation (4.18) is satisfied by $f(s)=\mathrm{e}^{-s}$ as well as by $f(s)=1 /(1+$ $s)^{1+k}$, for $k>1$.

Remark 4.3. In the case where $f(s)=1 /(1+s)^{1+k}$ for $0<k<1,(4.18)$ is not satisfied and in order to prove blow-up we may choose $\delta(M)=(1+M)^{2(1+q)}$ where $k>q>k / 2$. Then, following the same steps as in the general decreasing case we obtain that $\|u(\cdot, t)\|_{\infty} \rightarrow \infty$ as $t \rightarrow t^{*}$, where now

$$
t^{*} \lesssim \frac{(1+q)}{p\left(\lambda-\lambda^{*}\right) M^{p}(0)} \quad \text { for } p=2(k-q)
$$

Remark 4.4. Our construction of exploding lower solution $z(x, t)$ is still valid in the case of a bending bifurcation diagram considering again sufficiently large initial data. Indeed, we impose the condition that $M(t)$ satisfies

$$
\dot{M}(t):=\frac{\mathrm{d} M}{\mathrm{~d} t}=\frac{\lambda-\lambda_{*}}{\left(\int_{\Omega} f(z) \mathrm{d} x\right)^{2}} \inf _{x \in \Omega}\left\{\frac{f(z)}{z_{M}}\right\}>0 \quad \text { for } t>0
$$


for $\lambda_{*}<\lambda<\lambda^{*}$, where $\lambda_{*}=\lim _{s \rightarrow 0}(s)>0$. Consequently, we get

$$
\begin{aligned}
z_{t}-\Delta z-\frac{\lambda f(z)}{\left(\int_{\Omega} f(z) \mathrm{d} x\right)^{2}} & =z_{M} \dot{M}(t)-\Delta z-\frac{\lambda f(z)}{\left(\int_{\Omega} f(z) \mathrm{d} x\right)^{2}} \\
& \leqslant z_{M} \dot{M}(t)+\delta(t) f(z)-\frac{\lambda f(z)}{\left(\int_{\Omega} f(z) \mathrm{d} x\right)^{2}} \quad \text { due to (4.11) } \\
& \lesssim z_{M} \dot{M}(t)+\frac{\left[\lambda_{*}-\lambda\right] f(z)}{\left(\int_{\Omega} f(z) \mathrm{d} x\right)^{2}} \\
& =z_{M}\left(\frac{\lambda-\lambda_{*}}{\left(\int_{\Omega} f(z) \mathrm{d} x\right)^{2}} \inf _{x \in \Omega}\left\{\frac{f(z)}{z_{M}}\right\}+\frac{\left[\lambda_{*}-\lambda\right]}{\left(\int_{\Omega} f(z) \mathrm{d} x\right)^{2}} \frac{f(z)}{z_{M}}\right) \\
& =\frac{z_{M}\left(\lambda-\lambda_{*}\right)}{\left(\int_{\Omega} f(z) \mathrm{d} x\right)^{2}}\left[\inf _{x \in \Omega}\left\{\frac{f(z)}{z_{M}}\right\}-\frac{f(z)}{z_{M}}\right] \leqslant 0,
\end{aligned}
$$

since $\delta(t) \lesssim \lambda_{*} /\left(\int_{\Omega} f(z) \mathrm{d} x\right)^{2}$ holds for $M(0)$ sufficiently large, which results from $(*)$, (4.18) and the definition of $z(x, t)$. Following the same steps as above it can be proven that $M(t)$ blows-up in finite time

$$
t_{1}^{*} \lesssim \frac{|\Omega|^{2}}{\left(\lambda-\lambda_{*}\right)} \int_{M(0)}^{\infty}\left[2 \sigma f(\sigma)-\sigma^{2} f^{\prime}(\sigma)\right] \mathrm{d} \sigma<\infty,
$$

provided again that $f$ satisfies (4.18). Hence, it follows that $u(x, t)$ blows up globally and uniformly in finite time $t^{*} \leqslant t_{1}^{*}$.

Acknowledgements. The preparation of this article was supported by the EU network HYKE under the contract HPRN-CT-2002-00282. The work of N.I.K. started when he was visiting the Institute of Mathematics of Wrocław University and it was completed during his stay at the Faculty of Mathematics, Computer Science and Econometrics of the University of Zielona Góra. He thanks both of the institutes for their hospitality. N.I.K. also thanks Grzegorz Karch and Robert Stańczy for their fruitful observations and for suggesting references $[\mathbf{1 2}]$ and [15], respectively, and Andrew Lacey for his contribution to the improvement of this manuscript.

\section{References}

1. W. Allegretto And H. XIE, A non-local thermistor problem, Eur. J. Appl. Math. 6 (1995), 83-94.

2. H. Amann, Fixed point equations and nonlinear eigenvalue problems in ordered Banach spaces, SIAM Rev. 18 (1976), 620-709.

3. S. N. Antontsev And M. Chipot, The thermistor problem: existence, smoothness, uniqueness, blow-up, SIAM J. Math. Analysis 25(4) (1994), 1128-1156.

4. S. N. Antontsev And M. Chipot, The analysis of blow-up for the thermistor problem, Sb. Math. J. 38 (1997), 827-841.

5. A. Barabanova, The blow-up of solutions of a non-local thermistor problem, Appl. Math. Lett. 9 (1996), 59-63.

6. J. W. Bebernes And A. A. LaCey, Global existence and finite-time blow-up for a class of non-local parabolic problems, Adv. Diff. Eqns 2 (1997), 927-953. 
7. J. W. Bebernes And P. TAlaga, Non-local problems modelling shear banding, Commun. Appl. Nonlin. Analysis 3 (1996), 79-103.

8. J. W. Bebernes, C. Li and P. Talaga, Single-point blow-up for non-local parabolic problems, Physica D 134 (1999), 48-60.

9. E. Caglioti, P.-L. Lions, C. Marchioro and M. Pulvirenti, A special class of stationary flows for two-dimensinal Euler equations: a statistical mechanics description, Commun. Math. Phys. 143 (1992), 501-525.

10. G. Cimatti, Remark on existence and uniqueness for the thermistor problem under mixed boundary conditions, Q. Appl. Math. 47 (1989), 117-121.

11. G. Cimatti, The stationary thermistor problem with a current limiting device, Proc. $R$. Soc. Edinb. A 116 (1990), 79-84.

12. A. CONSTANTIN AND J. Escher, Wave breaking for nonlinear non-local shallow water equations, Acta Math. 181 (1998), 229-243.

13. J. Dieudonné, Foundations of modern analysis (Academic Press, 1969).

14. L. Evans And R. GARIEPy, Measure theory and fine properties of functions, Studies in Advanced Mathematics (CRC Press, Boca Raton, FL, 1992).

15. A. FRIEDman And B. MCLEOD, Blow-up of positive solutions of semilinear heat equations, Indiana Univ. Math. J. 34 (1985), 425-447.

16. B. Hu AND H. M. YIN, Semilinear parabolic equations with prescribed energy, Rend. Circ. Mat. Palermo 44(3) (1995), 479-505.

17. N. I. Kavallaris And D. E. Tzanetis, On the blow-up of a non-local parabolic problem, Appl. Math. Lett. 19 (2006), 921-925.

18. N. I. Kavallaris, C. V. Nikolopoulos and D. E. Tzanetis, Estimates of blow-up time for a non-local problem modelling an ohmic heating process, Eur. J. Appl. Math. 13 (2002), 337-351.

19. A. KRZYWiCki And T. NAdZIEJA, Some results concerning the Poisson-Boltzmann equation, Zastosowania Mat. (Appl. Math. (Warsaw)) 21 (1991), 265-272.

20. A. A. LACEY, Thermal runaway in a non-local problem modelling ohmic heating, Part I, Model derivation and some special cases, Eur. J. Appl. Math. 6 (1995), 127-144.

21. A. A. LACEY, Thermal runaway in a non-local problem modelling ohmic heating, Part II, General proof of blow-up and asymptotics of runaway, Eur. J. Appl. Math. 6 (1995), 201-224.

22. A. A. LaCey, Diffusion models with blow-up, J. Computat. Appl. Math. 97 (1998), 39-49.

23. D. E. Tzanetis, Blow-up of radially symmetric solutions of a non-local problem modelling ohmic heating, Electron. J. Diff. Eqns 11 (2002), 1-26.

24. G. WolAnsky, A critical parabolic estimate and application to non-local equations arising in chemotaxis, Applic. Analysis 66 (1997), 291-321. 